\title{
Expression of CXCL12 and CXCR4 in pT3-stage gastric cancer does not correlate with peritoneal metastasis
}

\author{
KENJI TSUBOI $^{1}$, YASUHIRO KODERA ${ }^{1}$, HAYAO NAKANISHI $^{2}$, SEIJI ITO ${ }^{3}$, YOSHINARI MOCHIZUKI ${ }^{3}$, \\ GORO NAKAYAMA ${ }^{1}$, MASAHIKO KOIKE ${ }^{1}$, MICHITAKA FUJIWARA ${ }^{1}$, \\ YOSHITAKA YAMAMURA ${ }^{3}$ and AKIMASA NAKAO ${ }^{1}$ \\ ${ }^{1}$ Department of Surgery II, Nagoya University Graduate School of Medicine, Aichi, Nagoya 466-8550; \\ ${ }^{2}$ Laboratory of Pathology, Aichi Cancer Center Research Institute; ${ }^{3}$ Department of \\ Gastroenterological Surgery, Aichi Cancer Center Hospital, Nagoya 464-8681, Japan
}

Received March 19, 2008; Accepted June 27, 2008

DOI: 10.3892/or_00000118

\begin{abstract}
CXCR4, a chemokine receptor, is considered to be involved in the metastastic formation of various types of cancer and could influence survival. More recently, CXCR4 was reported to be associated with peritoneal metastasis in gastric cancer, and CXCL12, its ligand, as a prognostic determinant among gastric cancer of various stages. In order to more specifically delineate the relevance of CXCR4 in peritoneal metastasis, 98 patients with pT3-stage gastric cancer who underwent gastrectomy and detection of intraabdominal free cancer cells in the peritoneal washing samples were evaluated. Immunostaining with anti-CXCL12 and anti-CXCR4 antibodies were performed for the primary tumor specimens, and correlation of the immunoreactivities with various clinicopathologic factors was evaluated. CXCR4 was detected in 61 specimens and CXCL12 in 76 specimens. No significant correlation was observed between presence of free cancer cells in the peritoneal cavity or development of clinical peritoneal carcinomatosis and expression of either the chemokine or the receptor. On the other hand, there was a trend towards correlation of expression of these molecules with recurrences to the distant lymph nodes or to the liver, although the number of events in these categories were insufficient to reach a statistical significance. In gastric cancer, CXCL12/ CXCR4 axis seems to be more strongly associated with lymphatic or hematogenous metastasis than the establishment of peritoneal deposits.
\end{abstract}

Correspondence to: Dr Yasuhiro Kodera, Department of Surgery II, Nagoya University Graduate School of Medicine, 65 Tsurumai-cho, Showa-ku, Aichi, Nagoya 466-8550, Japan

E-mail: ykodera@med.nagoya-u.ac.jp

Key words: chemokine, CXCR4, metastasis, gastric cancer, prognostic factor

\section{Introduction}

Gastric cancer is a common disease and remains a major health concern in the Far East as well as in other countries world wide (1). Prognosis of early-stage cancer is excellent, and various minimally invasive procedures have been exploited to dissect and cure these lesions (2). However, the outcome of advanced/metastatic disease remains dismal. Peritoneal carcinomatosis is the commonest pattern of failure when a goal of local control has been achieved through extended lymph node dissection $(3,4)$. The authors have explored peritoneal metastases from the viewpoint of free cancer cells shed in the abdominal cavity, which has been detected in $>65 \%$ of patients with serosa-positive gastric cancer (5). Some gastric cancers do not develop peritoneal metastases despite invasion of the serosal surface, however, suggesting that these cancers that develop peritoneal metastases are biologically different from those that do not. To improve treatment outcome of advanced gastric cancer, it is a prerequisite to be able to control the peritoneal metastasis. Attempts to elucidate the mechanisms underlining the organspecific metastatic capacity (6) of gastric cancer cells are therefore warranted.

CXCR4 is a key receptor in the cross talk between tumor cells and their microenvironment (7). Mesenchymal cells and other non-neoplastic stromal cells constitutively secrete the chemokine stromal cell-derived factor-1 (SDF-1/CXCL12) (8). In addition, CXCL12 is produced by various cancer cells and may act also in autocrine fashion $(9,10)$. The chemokine attracts circulating cells through its cognate receptor, CXCR4. The distinct pattern of chemokine receptor expression by tumor cells is considered to have a critical role in determining the site(s) of metastatic spread (6), and cancer cells that express CXCR4 are attracted to organs where CXCL12 is expressed (11). CXCL12 also induces migration, growth and survival of neoplastic cells in a paracrine fashion $(10,12)$, and promote tumor angiogenesis by attracting endothelial cells to the microenvironment (12). Thus, expression of CXCR4 is associated with metastatic formation, including micrometastasis (13), in various types of cancer and could consequently serve as a prognostic marker (14-21). Recently, 
CXCR4 was reported also to play an important role in the development of peritoneal metastasis from gastric carcinoma (22), while the expression of the chemokine CXCR12 in the primary cancer was reported to be an independent prognostic factor among gastric cancer patients of various stages (23). These reports prompted the authors to evaluate the expressions of CXCL12 and CXCR4 in a more specific data set consisting only of pT3-stage gastric cancer whose details regarding long-term outcome, occurrence of peritoneal carcinomatosis, and detection status of free cancer cells in the abdominal cavity are available.

\section{Patients and methods}

Patients. Ninety-eight consecutive patients with pT3-stage gastric cancer who underwent gastrectomy at Department of Gastroenterological Surgery, Aichi Cancer Center between June 1995 and June 2001 and underwent detection of intraabdominal free cancer cells form the basis of this study. All patients had been considered candidates for curative resection, but staging laparoscopy had not been performed as a part of preoperative work up. None of the patients had received preoperative chemotherapy. Detection of free cancer cells in the peritoneal washes was performed at the time of laparotomy both by the conventional cytologic examination with Papanicolaou and Giemsa staining and molecular detection through amplification of carcinoembryonic antigen (CEA) mRNA by reverse-trascriptase polymerase reaction (RT-PCR) (5). All the resected specimens were evaluated histopathologically after hematoxylin and eosin staining for accurate staging based on the Japanese Classification of Gastric Carcinoma (24). Patients were followed for over 5 years (median, 2059 days; range, 1832-3289 days) or until death.

Surgical procedure and postoperative surveillance of patients. After laparotomy, the abdominal cavity was thoroughly examined for tumor metastasis. Samples of the peritoneal deposits were usually taken for pathologic confirmation. Gastrectomy with D2 lymphadenectomy was the treatment of choice for potentially curable gastric cancer. The follow-up program consisted of interim history, physical examination, hematology and blood chemistry panels including tests for CEA and carbohydrate antigen 19-9, and computerized tomography performed at least once every 6 months. Peritoneal carcinomatosis was evident on the basis of clinical symptoms, digital examination, physical and radiological findings of bowel obstruction and ascites. Histological confirmation through biopsy and paracenthesis was performed where possible.

Immunohistochemistry. Histology and immunohistochemistry were performed on sections of $4 \mu \mathrm{m}$ thickness from formalinfixed, paraffin-embedded tissues dewaxed through xylene and graded concentrations of ethanol. For histology, sections were stained with hematoxylin and eosin (H\&E). For immunohistochemistry, sections were pretreated for antigen retrieval by microwave at $98^{\circ} \mathrm{C}$ for $10 \mathrm{~min}(\mathrm{pH} \mathrm{6.0)}$ for CXCR4 and CXCL12. These sections were immersed in methanol with $0.3 \%$ hydrogen peroxide for $20 \mathrm{~min}$ to inactivate endogenous peroxidase activity, followed by normal horse serum for $30 \mathrm{~min}$ to block non-specific reactions.
Sections were incubated at $4^{\circ} \mathrm{C}$ overnight with the following first antibodies diluted with PBS containing 1\% BSA; mouse monoclonal antibodies against human CXCR4 (1:5000 dilution, R\&D Systems, Minneapolis, MN) and CXCL12 (1:500 dilution, R\&D Systems). After washing with PBS, sections were incubated with biotinylated second antibody for $30 \mathrm{~min}$. Sections were washed again with PBS, then incubated with streptavidin-peroxidase complex (Vectastain ABC kit; Vector, Burlingame, CA) for $60 \mathrm{~min}$. Chromogen was developed with $0.01 \%$ diaminobenzidine, and sections were counterstained with hematoxylin. A pathologist (H.N.) who had no information regarding the outcome of patients evaluated the sections using a scoring system based on percentage of positively stained cells $(0,<10 \%$ positive cells; $1+, 10-50 \%$ positive cells; $2+,>50 \%$ positive cells) according to a cut-off value by Sato et al (25). The sections were graded as negative (0) and positive (1+ and $2+)$.

Collection of the peritoneal washes and real-time RT-PCR. Just after laparotomy, $100 \mathrm{ml}$ of saline were introduced into the Douglas and left subphrenic cavities and aspirated after gentle stirring, centrifuged at $1800 \mathrm{rpm}$ for $5 \mathrm{~min}$ to collect intact cells, rinsed with phosphate buffer saline, dissolved in Isogen RNA extraction buffer (Nippon Gene, Tokyo, Japan) and stored at $-80^{\circ} \mathrm{C}$ until use. A portion of each peritoneal washing sample underwent cytologic examination using conventional Papanicolaou and Giemsa staining.

Real-time RT-PCR detection of CEA and GAPDH (glyceraldehyde-3-phosphate dehydrogenase) mRNA in the peritoneal washing samples was performed as described elsewhere (5). In brief, total RNA was extracted using a guanidinium isothiocyanate-phenol-chloroform method. Extracted total RNA was converted to first-strand cDNA and was immediately used for PCR amplification with a LightCycler (Roche Diagnostics, Mannheim, Germany). Real-time RT-PCR was performed by a single-step method (50 cycles) using hybridization probes. Design of the primers and probes used in the study, together with the conditions for amplification, were described previously (5). All the primers and probes were synthesized and purified by reverse-phase high-performance liquid chromatography by Nihon Gene Research Laboratories (Sendai, Japan). Real-time PCR monitoring was achieved by measuring the fluorescent signal at the end of the annealing phase for each cycle. GAPDH was quantified only to ensure that mRNA was successfully extracted. A cut-off value of 0.1 for CEA mRNA (equivalent to one-tenth of CEA mRNA contained in a single COLM-2 cell) was established by the aid of a receiver operating characteristics curve which was constructed using data for CEA mRNA values and presence or absence of free intraabdominal cancer cells (presence of cancer cells in this analysis was defined as either having peritoneal metastasis at surgery or suffering from relapse as peritoneal carcinomatosis within 2 years of surgery), as reported previously (5). For individuals with samples from both Douglas and left subphrenic cavities, the higher value of CEA mRNA was used for the analysis.

Statistical analysis. Survival curves were constructed by the Kaplan-Meier method and Log-rank test was used to evaluate 


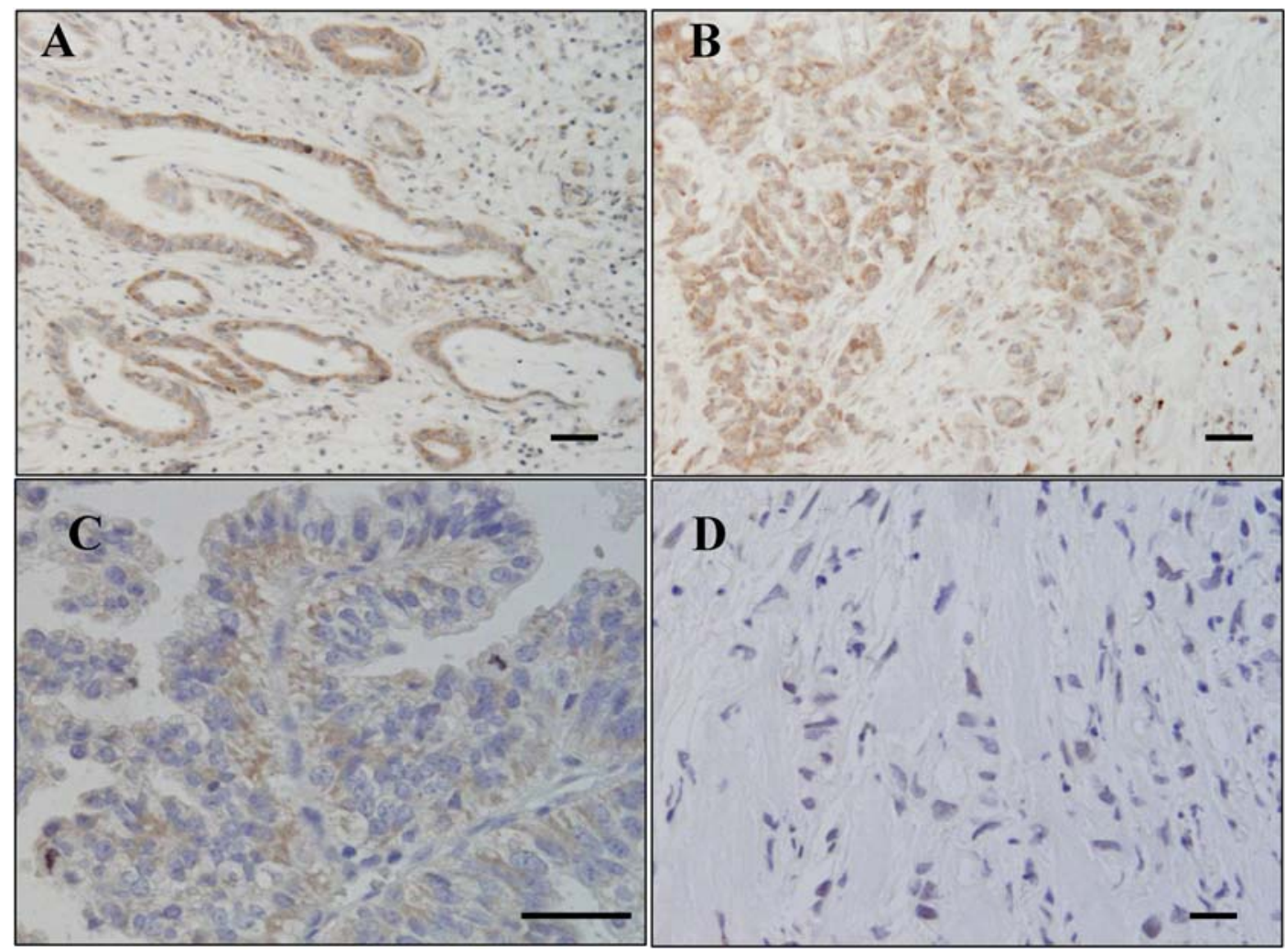

Figure 1. Immunohistochemical analysis of CXCR4 expression in the primary gastric tumor and metastasis. (A) Differentiated type adenocarcinoma stained for CXCR4 at the invasion front in the primary tumor. (B) Poorly-differentiated type adenocarcinoma stained for CXCR4 at the invasion front. (C) Metastatic liver tumor weakly stained for CXCR4. (D) Metastatic tumor in the peritoneum negatively stained for CXCR4. Bars, $100 \mu \mathrm{M}$.

the differences between the curves. $\chi^{2}$ test was performed to evaluate correlation between the expression of CXCL12 or CXCR4 and various clinicopathologic factors and patterns of disease failure.

\section{Results}

Patient demographics. Mean age of the patients was $60.3 \pm 12.2$ with the male:female ratio of 59:39. Node metastasis was frequent, and only 8 patients were found after systemic lymphadenectomy to have no lymph node metastasis. Despite the preoperative diagnosis through conventional imaging studies that these patients have no distant metastasis, peritoneal deposits were found at laparotomy in as many as 29 patients (30\%). Cytologic examination was positive in 31 patients (32\%) and CEA RT-PCR in 68 patients (76\%).

Outcome and patterns of disease failure. There was no operative mortality. Median survival of all patients was 585 days. Seventy-six of 98 patients had recurrences during the follow-up. Site of recurrence was peritoneal in 60 patients (61\% of all patients, $80 \%$ of all patients with recurrent disease), nodal in 19 patients, hepatic in 9 patients, bone in 8 patients and other sites in 4 patients.

Expression of CXCR4 and CXCL12. CXCR4 was detected in 61 specimens $(1+$ in 49 specimens and $2+$ in 12$)$, and CXCL12 in 76 specimens ( $1+$ in 30 specimens and $2+$ in 46 ). Little difference in clinicopathologic factors were observed between the tumors with $1+$ status and those with $2+$ status, and these were treated collectively in the following analyses as positive for CXCR4 or CXCL12. Tumor cells were stained for CXCR4 moderately in the cytoplasm and weakly in the cell membrane in both the differentiated and poorly differentiated types (Fig. 1A and B). Staining of CXCR4 was inclined to be more prominent in the invasion front. CXCL12 was strongly stained in the cytoplasm of gastric cancer cells in both the differentiated and poorly-differentiated types (Fig. 2A and B). Staining of CXCL12 seemed to be more intense in floating tumor cells in the lymphatic vessels (Fig. 2C).

Correlations of CXCR4 and CXCL12 expression with various clinicopathologic variables and types of disease failure are summarized in Tables I and II. No significant correlation was observed between presence of free cancer cells in the peritoneal cavity or development of peritoneal carcinomatosis and expression profile of the CXCL12/CXCR4 axis. On the other hand, there was a trend towards correlation of the immunoreactivity to CXCR4 with recurrences to the distant lymph nodes $(\mathrm{p}=0.1588)$, and hematogenously to the liver or the bone $(p=0.0971)$, although the number of events was too small to reach a statistical significance. A section of liver metastasis was available in 1 of 9 patients who had hepatic metastasis or recurrence. Cancer cells in this specimen were positively stained both with CXCR4 and CXCL12 (Figs. 1C and 2D). At the same time, intrahepatic bile duct and portal vein in the non-cancerous liver was positively stained (Fig. 2E). Immunostaining was attempted also for some samples of peritoneal seeding, but CXCR4 was rarely stained (Fig. 1D) 

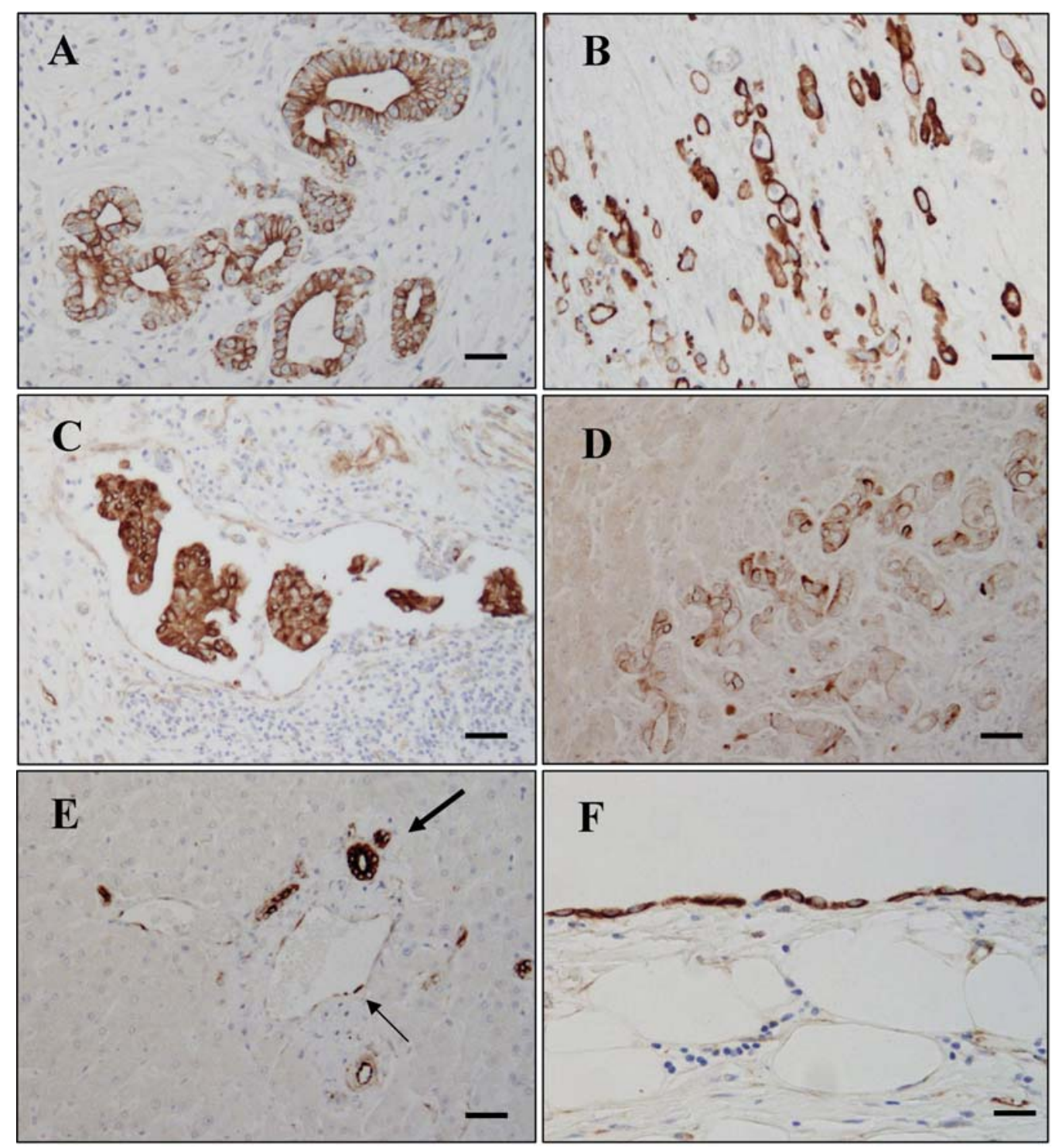

Figure 2. Immunohistochemical analysis of CXCL12 expression in the primary gastric tumor and metastasis. (A) Differentiated type adenocarcinoma stained for CXCL12 in the primary tumor. (B) Poorly-differentiated type adenocarcinoma stained for CXCL12 in the primary tumor. (C) Tumor cells permeated into the lymphatic vessels strongly stained for CXCL12 in the primary tumor. (D) Metastatic liver tumor moderately stained for CXCL12. (E) Non-cancerous tissue in the metastatic liver foci. Intrahepatic bile duct epithelial cells (thick arrow) and portal vein endothelial cells (thin arrow) was stained positive. (F) Mesothelial lining cells also stained positive for CXCL12. Bars, $100 \mu \mathrm{M}$.

despite the strong expression of CXCL12 observed in the mesothelial cells lining the peritoneum (Fig. 2F). CXCR4 was less frequently expressed in linitis plastica type gastric cancer as opposed to the other types $(\mathrm{p}=0.0412)$, and had a weak tendency to be detected more frequently among the differentiated phenotype $(\mathrm{p}=0.2890)$. The expression of CXCR4 or CXCL12 had no influence on survival of pT3stage cancers, even when the comparison was made between $\mathrm{CXCR} 4^{+} \mathrm{CXCL} 12^{+}$patients and $\mathrm{CXCR} 4{ }^{-} \mathrm{CXCL} 12^{-}$patients.

\section{Discussion}

Peritoneal metastasis is a prominent pattern of disease failure observed frequently among serosa-positive (T3 or T4 stage) gastric cancer $(3,4)$. It is considered to occur when cancer cells are exfoliated from the serosal surface and attach to any part of the peritoneal lining where it can proliferate. Detection of free cancer cells in the peritoneal washes is considered to be highly predictive of developing peritoneal disease (26), and even minimal disease detected by RT-PCR affects outcome of a patient substantially (5). This is in sharp contrast with minimal residual disease within the lymph nodes which was shown in an in vivo model to regress spontaneously through resection of the primary tumor (27). Consequently, detection of nodal micrometastases through immunostaining in surgically resected specimens does not seem to affect the outcome of patients treated with systematic lymph node dissection (28).

One can speculate that floating in the peritoneal cavity resembles laboratory cell culture condition which is apparently a friendlier environment when compared with striving for survival in a lymph node packed with lymphocytes. Thus, a minute quantity of cancer cells floating in the abdominal cavity can influence survival and, according to the current study, activation of the CXCL12-CXCR4 axis is not necessarily essential for the establishment of peritoneal 
Table I. Correlation of the expression of CXCR4 with clinicopathologic variables.

\begin{tabular}{|c|c|c|c|c|c|c|c|}
\hline CXCR4 & $(-)$ & $(+)$ & P-value & CXCL12 & $(-)$ & $(+)$ & P-value \\
\hline Gender & & & & Gender & & & \\
\hline Male & 19 & 40 & & Male & 14 & 45 & \\
\hline Female & 18 & 21 & 0.2374 & Female & 8 & 31 & 0.8996 \\
\hline Age & & & & Age & & & \\
\hline$<50$ & 6 & 13 & & $<50$ & 4 & 15 & \\
\hline $50-69$ & 19 & 37 & & $50-69$ & 12 & 44 & \\
\hline$\geq 70$ & 12 & 11 & 0.2603 & $\geq 70$ & 6 & 17 & 0.8915 \\
\hline Histology & & & & Histology & & & \\
\hline Differentiated & 5 & 15 & & Differentiated & 3 & 17 & \\
\hline Undifferentiated & 32 & 46 & 0.2890 & Undifferentiated & 19 & 59 & 0.5521 \\
\hline Node metastasis $(\mathrm{pN})$ & & & & Node metastasis (pN) & & & \\
\hline Negative & 4 & 4 & & Negative & 2 & 6 & \\
\hline Positive & 32 & 56 & 0.7029 & Positive & 20 & 68 & $>0.9999$ \\
\hline Concomitant peritoneal seeding & & & & Concomitant peritoneal seeding & & & \\
\hline Negative & 25 & 44 & & Negative & 14 & 55 & \\
\hline Positive & 12 & 17 & 0.8014 & Positive & 8 & 21 & 0.5996 \\
\hline Cytology of the peritoneal washes & & & & Cytology of the peritoneal washes & & & \\
\hline Negative & 27 & 40 & & Negative & 14 & 53 & \\
\hline Positive & 10 & 21 & 0.5895 & Positive & 8 & 23 & 0.7783 \\
\hline CEA RT-PCR & & & & CEA RT-PCR & & & \\
\hline Negative & 11 & 19 & & Negative & 9 & 21 & \\
\hline Positive & 26 & 42 & $>0.9999$ & Positive & 13 & 55 & 0.3538 \\
\hline Linitis plastica & & & & Linitis plastica & & & \\
\hline No & 19 & 45 & & No & 11 & 53 & 0.1447 \\
\hline Yes & 18 & 16 & 0.0412 & Yes & 11 & 23 & \\
\hline Recurrence as peritoneal disease & & & & Recurrence as peritoneal disease & & & \\
\hline No & 15 & 23 & & No & 10 & 12 & \\
\hline Yes & 22 & 38 & 0.9478 & Yes & 28 & 48 & 0.6300 \\
\hline Recurrence in the lymph nodes & & & & Recurrence in the lymph nodes & & & \\
\hline No & 33 & 46 & & No & 20 & 59 & \\
\hline Yes & 4 & 15 & 0.1588 & Yes & 2 & 17 & 0.2797 \\
\hline Recurrence in the liver & & & & Recurrence in the liver & & & \\
\hline No & 36 & 53 & & No & 21 & 68 & \\
\hline Yes & 1 & 8 & 0.1709 & Yes & 1 & 8 & 0.6626 \\
\hline Recurrence as bone metastasis & & & & Recurrence as bone metastasis & & & \\
\hline No & 35 & 55 & & No & 21 & 69 & \\
\hline Yes & 2 & 6 & 0.6921 & Yes & 1 & 7 & 0.7936 \\
\hline Hematogenous metastasis & & & & Hematogenous metastasis & & & \\
\hline No & 35 & 49 & & No & 20 & 64 & \\
\hline Yes & 2 & 12 & 0.0971 & Yes & 2 & 12 & 0.6565 \\
\hline
\end{tabular}

Table II. Correlation of the expression of CXCL12 with clinicopathologic variables.

be attracted. However, free cancer cells can land on the peritoneal surface without being attracted, and firm adhesion followed by transendothelial migration into tissues essential metastasis. Mesothelial cells lining the peritoneum areknown to express CXCL12, to which CXCR4-positive cancer cells that have been shed from the serosa-positive primary could 
for establishment of metastasis could then be governed by other molecules such as integrins and selectins (29). Yasumoto et al reported on a significant correlation between CXCR4 expression in the primary tumor and peritoneal carcinomatosis by immunostaining 29 cases with stage IV gastric cancer (22). However, their data that 11 of 23 patients (49\%) with CXCR4-negative cancer suffered from peritoneal carcinomatosis still point to the fact that CXCR4 expression in the primary tumor may not be an essential factor in the formation of peritoneal metastasis.

Chemokine gradients may have a greater role in transient and reversible interaction between circulating tumor cells and vascular endothelium in appropriate organs where CXCL12 is constitutively expressed by stromal fibroblasts. Role of CXCL12/CXCR4 axis in bone metastasis of breast cancer (13) or liver metastasis of colorectal cancer (18) may apply also to gastric cancer. In the current study, primary tumor of 8 of 9 patients with hepatic metastasis and 6 of 8 patients with bone metastasis were positively stained for CXCR4, although these tendencies did not reach a statistical significance due possibly to the small number of events. Linitis plastica type cancer represents a peculiar subset of gastric cancer which often metastasizes to the peritoneal lining but is rarely seen harboring the liver (30). In the current study, expression of CXCR4 was significantly less frequent among linitis plastica type cancers. None of 34 patients with this type of cancer had hepatic metastasis, whereas 3 of 16 linitis plastica type that were positive for CXCR4 had metastasis to the bone. Despite the relatively low positive rate of CXCR4 expression in the linitis plastica in comparison with other types of gastric cancer, gross peritoneal deposits were observed in 11 patients (32\%) at surgery, and peritoneal carcinomatosis eventually developed in as many as 25 patients $(74 \%)$. This suggests again that CXCL12/CXCR4 axis does not play a major role in peritoneal metastasis.

Interpretation of the chemokine CXCL12 expressed by cancer cells themselves is more perplexing, given the general understanding that chemokines may play an important role in organ specificity of metastasis (6). Since chemokines are known to stimulate growth of cancer cells and promote angiogenesis (12), chemokines secreted by cancer cells may enhance their proliferation in an autocrine fashion while promoting angiogenesis in their microenvironment. Ishigami et al reported through a study employing immunostaining that the expression of CXCL12 influences outcome of patients with gastric cancer (23). In their study, the positivity rate of CXCL12 staining was elevated as the pT stage increased (positivity had been $18 \%$ for pT1, 54\% for pT2 and $63 \%$ for T3), meaning that higher clinical stages may have been at least partially responsible for the inferior prognosis of CXCL12-positive gastric cancer. Perhaps, this difference in survival was not observed in the current study since all samples tested had been pT3 stage cancer in which CXCL12 was positively stained in as many as 76 of 98 patients (78\%).

Neither was the expectation that CXCR4 staining could serve as a prognostic determinant fulfilled in this study. Although CXCR4 may have a role in hematogenous metastasis, it may not have influenced the outcome significantly since hematogenous metastasis is not a common pattern of disease failure among patients with pT3-stage gastric cancer. Surprisingly, Kwak et al reported through another immunostaining study that prognosis of CXCR4positive gastric cancers was in fact superior to the CXCR4negative counterparts (31). The only finding in common with the current study was that CXCR4 was more frequently expressed among the differentiated phenotype.

To conclude, although it is not possible to deny the role of CXCL12/CXCR4 axis in the peritoneal carcinomatosis of gastric cancer, it seems to be more strongly associated with hematogenous metastasis. Immunostaining of these molecules is not currently useful as a prognostic determinant of pT3stage cancer. Further in vitro studies are required to elucidate the role of CXCL12 production by cancer cells.

\section{References}

1. Pisani P, Parkin DM, Bray F and Ferlay J: Estimates of the worldwide mortality from 25 cancers in 1990. Int J Cancer 83: 18-29, 1999.

2. Yokota T, Ishiyama S, Saito T, Teshima S, Shimotsuma M and Yamauchi H: Treatment strategy of limited surgery in the treatment guidelines for gastric cancer in Japan. Lancet Oncol 4: 423-428, 2003.

3. Maehara Y, Hasuda S, Koga T, Tokunaga E, Kakeji Y and Sugimachi K: Postoperative outcome and sites of recurrence in patients following curative resection of gastric cancer. Br J Surg 87: 353-357, 2000.

4. Yoo CH, Noh SH, Shin DW, Choi SH and Min JS: Recurrence following curative resection for gastric carcinoma. Br J Surg 87: 236-242, 2000.

5. Kodera Y, Nakanishi H, Ito S, et al: Prognostic significance of intraperitoneal cancer cells in gastric carcinoma: analysis of real-time RT-PCR after 5 years of follow-up. J Am Coll Surg 212: 231-236, 2006.

6. Ben-Baruch A: Organ selectivity in metastasis: regulation by chemokines and their receptors. Clin Exp Metastasis 25: 345356, 2008.

7. Burger JA and Kipps TJ: CXCR4: a key receptor in the cross talk between tumor cells and their microenvironment. Blood 107: 1761-1767, 2006.

8. Oonakahara K, Matsuyama W, Higashimoto I, Kawabata M, Arimura K and Osame M: Stromal-derived factor-1 $\alpha /$ CXCL12CXCR4 axis is involved in the dissemination of NSCLC cells into pleural space. Am J Respir Cell Mol Biol 30: 671-677, 2004.

9. Scotton CJ, Wilson JL, Scorr K, et al: Multiple actions of the chemokine CXCL12 on epithelial tumor cells in human ovarian cancer. Cancer Res 62: 5930-5938, 2002.

10. Koshiba T, Hosotani R, Miyamoto Y, et al: Expression of stromal cell-derived factor I and CXCR4 ligand receptor system in pancreatic cancer: a possible role for tumor progression. Clin Cancer Res 6: 3530-3535, 2000.

11. Muller A, Homey B, Soto H, et al: Involvement of chemokine receptors in breast cancer metastasis. Nature 410: 50-56, 2001.

12. Orimo A, Gupta PB, Sgroi DC, et al: Stromal fibroblasts present in invasive human breast carcinomas promote tumor growth and angiogenesis through elevated SDF-1/CXCL12 secretion. Cell 121: 335-348, 2005

13. Cabioglu N, Sahin A, Doucet M, et al: Chemokine receptor CXCR4 expression in breast cancer as a potential predictive marker of isolated tumor cells in bone marrow. Clin Exp Med 22: 39-46, 2005.

14. Muller A, Sonkoly E, Eulert C, et al: Chemokine receptors in head and neck cancer: association with metastatic spread and regulation during chemotherapy. Int J Cancer 118: 2147-2157, 2006.

15. Marchesi F, Monti P, Leone BE, et al: Increased survival, proliferation, and migration in metastatic human pancreatic tumor cells expressing functional CXCR4. Cancer Res 64: 8420-8427, 2004.

16. Burger M, Glodeck A, Hartmann T, et al: Functional expression of CXCR4 (CD184) on small-cell lung cancer cells mediates migration, integrin activation, and adhesion to stromal cells. Oncogene 22: 8093-8101, 2003. 
17. Scala S, Ottaiano A, Ascierto PA, et al: Expression of CXCR4 predicts poor prognosis in patients with malignant melanoma. Clin Cancer Res 11: 1835-1841, 2005.

18. Kim J, Mori T, Chen SL, et al: Chemokine receptor CXCR4 expression in patients with melanoma and colorectal cancer liver metastases and the association with disease outcome. Ann Surg 244: 113-120, 2006.

19. Schimanski CC, Schwald S, Simiantonaki N, et al: Effect of chemokine receptors CXCR4 and CCR7 on the metastatic behavior of human colorectal cancer. Clin Cancer Res 11: $1743-1750,2005$

20. Su L, Zhang J, Xu H, Wang Y, Chu Y, Liu R and Xiong S: Differential expression of CXCR 4 is associated with the metastatic potential of human non-small cell lung cancer cells. Clin Cancer Res 11: 8273-8280, 2005.

21. Kaifi JT, Yelebas EF, Schurr P, et al: Tumor-cell homing to lymph nodes and bone marrow and CXCR4 expression in esophageal cancer. J Natl Cancer Inst 97: 1840-1847, 2005.

22. Yasumoto K, Koizumi K, Kawashima A, et al: Role of the CXCL12/CXCR4 axis in peritoneal carcinomatosis of gastric cancer. Cancer Res 66: 2181-2187, 2006.

23. Ishigami $\mathrm{S}$, Natsugoe $\mathrm{S}$, Okumura $\mathrm{H}$, et al: Clinical implication of CXCL12 expression in gastric cancer. Ann Surg Oncol 14: 3154-3158, 2007.

24. Japan Gastric Cancer Association: Japanese Classification of Gastric Carcinoma, 2nd English edition. Gastric Cancer 1: 10-24, 1998.
25. Sato T, Murakumo Y, Hagiwara S, Jijiwa M, Suzuki C, Yatabe Y and Takahashi M: High-level expression of CD109 is frequently detected in lung squamous cell carcinomas. Pathol Int 57: 719-724, 2007.

26. Kodera Y, Yamamura Y, Shimizu Y, et al: Peritoneal washing cytology: prognostic value of positive findings in patients with gastric carcinoma undergoing a potentially curative resection. J Surg Oncol 72: 60-64, 1999.

27. Yokoyama H, Nakanishi H, Kodera Y, et al: Biological significance of isplated tumor cells and micrometastasis in lymph nodes evaluated using a green fluorescent protein-tagged human gastric cancer cell line. Clin Cancer Res 12: 361-368, 2006.

28. Fukagawa T, Sasako M, Mann GB, et al: Immunohistochemically detected micrometastases of the lymph nodes in patients with gastric carcinoma. Cancer 92: 753-760, 2001

29. Orr FW, Wang HH, Lafrenie RM, Scherbarth S and Nance DM: Interactions between cancer cells and the endothelium in metastasis. J Pathol 190: 310-329, 2000.

30. Kodera Y, Yamamura Y, Ito S, et al: Is Borrmann type IV gastric carcinoma a surgical disease? An old question revisited with reference to the result of peritoneal washing cytology. J Surg Oncol 78: 175-181, 2001

31. Kwak MK, Hur K, Park DJ, et al: Expression of chemokine receptors in human gastric cancer. Tumor Biol 26: 65-70, 2005 